Onkologe 2012 · 18:1070-1072

DOI 10.1007/s00761-012-2355-2

Online publiziert: 21. November 2012

(c) Springer-Verlag Berlin Heidelberg 2012

A. Hochhaus ${ }^{1} \cdot$ M. Freund ${ }^{2} \cdot$ R.-D. Kortmann ${ }^{3} \cdot$ K. Höffken ${ }^{4}$

${ }^{1}$ Klinik für Innere Medizin II, Abt. Hämatologie/Onkologie, Universitätsklinikum Jena

2 Zentrum für Innere Medizin, Klinik für Hämatologie, Onkologie und Palliativmedizin, Rostock

${ }^{3}$ Klinik für Strahlentherapie und Radioonkologie, Universitätsklinikum Leipzig

${ }^{4}$ Universitätsklinikum Jena

\title{
Leukämien als
} Modellerkrankungen für individualisierte Therapien

der Alterspyramide der Bevölkerung in Richtung auf ein höheres Lebensalter und durch die jüngsten Therapieerfolge die Prävalenz der Leukämiepatienten in den kommenden Jahren steigen.

Systematische Therapieoptimierungsstudien begannen in den 1980er Jahren mit der Gründung von Studiengruppen für die AML, die akute lymphatische Leukämie (ALL) und die chronische myeloische Leukämie (CML), die damals aus Bundesmitteln gefördert wurden. Diese akademischen Studiengruppen haben während der vergangenen 30 Jahre wesentlich zur Verbesserung der Versorgung der Bevölkerung und zur Förderung einer innovativen, interdisziplinären Leukämieforschung beigetragen. Umfangreiche Begleitforschungsprogramme haben das Verständnis über die Biologie der Leukämien verbessert. Gerade durch diese Studien hat Deutschland in der Welt eine führende Position auf dem Gebiet der Hämatologie erlangt. Die Novellierung des Arzneimittelgesetzes im Jahre 2004 hat die Durchführung von industrieunabhängigen Therapiestudien und somit diese wichtige standardisierte Qualitätskontrolle der klinischen Forschung leider erheblich erschwert und indirekt industrieinitiierte Studien gefördert.

Meilensteine der kooperativen klinischen Forschung in Deutschland und Europa waren die Etablierung des Kompetenznetzes „Akute und chronische Leukämien“ im Jahre 1999 und die Gründung des European LeukemiaNet im Januar 2004.

\) Leukämien dienen als Modell

für neoplastische, genetische und infektiöse Erkrankungen

Leukämien dienen als Modell für eine Vielzahl anderer Krankheiten, wie neoplastische, genetische und infektiöse Erkrankungen. So stellen sie Modelle für initial disseminierte Erkrankungen dar, die allein mit Chemotherapie heilbar sind. In Bezug auf unser Pathogeneseverständnis, die Entwicklung innovativer Therapieansätze und neuer diagnostischer Verfahren wurde Pionierarbeit geleistet. Beispiel hierfür ist die CML als erste neoplastische Erkrankung, die mit einem erworbenen Chromosomendefekt (PhiladelphiaChromosom) assoziiert wurde, und mittlerweile die molekular am besten charakterisierte und therapierbare maligne Erkrankung darstellt. 88\% der mit Imatinib behandelten Patienten sind nach 8 Jahren noch am Leben. Die Progressionsrate der Erkrankung und die Geschwindigkeit molekularer Remissionen konnte durch die Zweitgenerationsinhibitoren Nilotinib und Dasatinib weiter verbessert werden, sodass heute im Rahmen klinischer Studien das Ziel der Heilung untersucht wird. Klar definierte zeitabhängige Meilensteine des Ansprechens erleichtern die Auswahl der optimalen Therapieoption. 
Hier steht eine Anzeige.

黛 Springer 
Auf individuelle molekulare Ziele der jeweiligen Tumorerkrankung ausgerichtete Therapien fanden nicht nur Eingang in die Routinebehandlung hämatologischer Erkrankungen. Auch bei soliden Tumoren konnten durch eine verbesserte Erkenntnis der essenziellen Signalwege der Tumorzellen wirksame Medikamente entwickelt und gezielt eingesetzt werden.

Das vorliegende Schwerpunktheft zeichnet die oben beschriebene Entwicklung bei Leukämien, Myelodysplasien und CMPN nach und fasst den neuesten Stand der biologischen, diagnostischen und therapeutischen Kenntnisse bei diesen Erkrankungen zusammen. Die Arbeitsgruppen aus München, Mannheim, Köln, Frankfurt, Dresden und Jena geben dem Leser einen umfassenden Überblick über aktuelle Daten und Entwicklungen.

So wird einerseits das allgemeine Prinzip der individualisierten Therapie in der Besonderheit der malignen hämatologischen Systemerkrankungen deutlich, andererseits aber auch die Dynamik in der Erforschung und deren Erfolge in der Bekämpfung der Leukämien erkennbar. Leukämien werden auch in den nächsten Jahrzehnten Modellerkrankungen für die Fortschritte in der Onkologie bleiben.

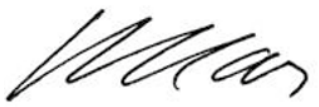

A. Hochhaus

Für die Herausgeber des Schwerpunkthefts

\section{ia. tof tren}

K. Höffken

Für die Herausgeber

\section{Korrespondenzadresse}

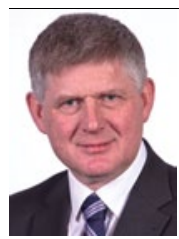

Prof. Dr. A. Hochhaus

Klinik für Innere Medizin II, Abt. Hämatologie/Onkologie, Universitätsklinikum Jena Erlanger Allee 101, 07740 Jena andreas.hochhaus@ med.uni-jena.de

\section{Ärzte Zeitung digital: Heute lesen, was morgen in der Zeitung steht}

\section{Im Publikumsbereich erfreuen sich App-Ausgaben von Zeitungen und Zeit- schriften wachsender Beliebtheit. Ab sofort ist auch Deutschlands einzige Ta- geszeitung für Ärzte in diesem Format erhältlich: die „Ärzte Zeitung digital“"}

„Ärzte Zeitung digital" bietet gesundheitspolitische Nachrichten, Kommentare, Hintergründe zu Medizin und wirtschaftlichen Themen rund um die Praxis - im übersichtlichen und intuitiven Lesemodus, optimiert für den Tablet-Computer. Die Vorteile für Leser der AppAusgabe der "Ärzte Zeitung":

- Die Ausgabe ist schon am Vorabend verfügbar, Leser können damit die Nachrichten des kommenden Tages bequem auf dem Sofa empfangen.

- Wichtige Nachrichten werden als „Breaking News" direkt auf das Tablet geleitet und lassen sich über die sogenannte Newslasche anzeigen

- Die App ist so konzipiert, dass die gesamte Ausgabe im Hintergrund heruntergeladen werden kann. Anders als im Internet ist es für Leser dann möglich, sich offline durch die Ausgabe zu „blättern“, ohne Wartezeiten beim Durchklicken auf einer Website.

- Die alten Ausgaben bleiben zwei Wochen auf dem Tablet-PC verfügbar.

„Ärzte Zeitung digital“ ist Teil des e.Med-Pakets von Springer Medizin. Zugänglich ist die App über den iTunes-Store von Apple - allerdings nur für registrierte Nutzer von Springer Medizin, die e.Med für 30 Tage kostenlos testen wollen, oder für e.Med-Abonnenten - und zwar ohne weitere Zusatzkosten. Die Version für Android-Tablets wird Anfang 2013 verfügbar sein.

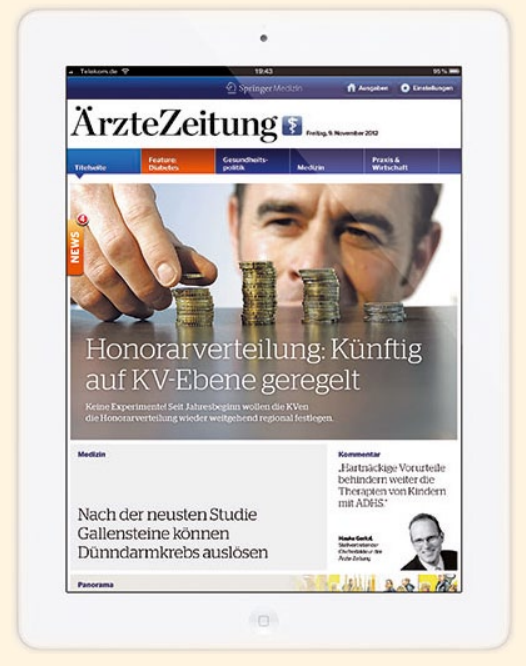

Abonnenten des e.Med-Pakets haben Zugriff auf rund 500 Fortbildungskurse in der e.Akademie, auf die Volltexte von mehr als 400 deutschsprachigen und internationalen Zeitschriften in der e.Bibliothek, auf einen Webbaukasten für die Praxiswebsite und ein PrintAbo einer Springer-Fachzeitschrift nach Wahl (plus Versandkostenpauschale). Das e.Med-Paket kostet 33,25 Euro im Monat.

Anmeldung zum kostenlosen e.Med-Testzugang: www.springermedizin.de/eMed/

Mehr Info zur App „Ärzte Zeitung digital“: www.springermedizin.de/tablet

Quelle: Ärzte Zeitung 